\title{
Cholinergic blockade inhibits gastro-oesophageal reflux and transient lower oesophageal sphincter relaxation through a central mechanism
}

\author{
J C Fang, I Sarosiek, Y Yamamoto, J Liu, R K Mittal
}

\begin{abstract}
Background-Atropine, an anticholinergic agent with central and peripheral actions, reduces gastro-oesophageal reflux (GOR) in normal subjects and patients with gastro-oesophageal reflux disease (GORD) by inhibiting the frequency of transient lower oesophageal sphincter relaxation (TLOSR).

Aims-To compare the effect of methscopolamine bromide (MSB), a peripherally acting anticholinergic agent, with atropine on the rate and mechanism of GOR in patients with GORD.

Methods-Oesophageal motility and $\mathrm{pH}$ were recorded for 120 minutes in 10 patients with GORD who were studied on three separate occasions. For the first two recording periods, either atropine (15 $\mu \mathrm{g} / \mathrm{kg}$ bolus, $4 \mu \mathrm{g} / \mathrm{kg} / \mathrm{h}$ infusion) or saline were infused intravenously. MSB (5 mg orally, four times daily) was given for three days prior to the third recording period.

Results-Atropine significantly reduced basal LOS pressure $(12.6(0.17) \mathbf{m m ~ H g}$ to $7.9(0.17) \mathrm{mm} \mathrm{Hg}$ ), and the number of TLOSR (8.1 (0.56) to $2.8(0.55))$ and reflux episodes $(7.0(0.63)$ to $2.0(0.43))(p<0.005$ for all comparisons). MSB reduced basal LOS pressure (12.6 (0.17) to $8.7(0.15) \mathrm{mm}$ $\mathrm{Hg}, \mathrm{p}<0.005)$, but had no effect on the frequency of TLOSR $(8.1(0.56)$ to $7.5(0.59))$ and reflux episodes $(7.0(0.63)$ to 4.9 $(0.60))(p>0.05)$.
\end{abstract}

Conclusion-In contrast to atropine, MSB has no effect on the rate of TLOSR or GOR in patients with GORD. Atropine induced inhibition of TLOSR and GOR is most likely mediated through a central cholinergic blockade.

(Gut 1999;44:603-607)

Keywords: gastro-oesophageal reflux; cholinergic blockade; transient lower oesophageal sphincter relaxation

Tonic contraction at the oesophagogastric junction is believed to be the principal mechanism preventing gastro-oesophageal reflux (GOR). The sphincter mechanism at the oesophagogastric junction is composed of the smooth muscle lower oesophageal sphincter (LOS) and the skeletal muscle crural diaphragm. Weakness of either can lead to GOR; however, the most prevalent mechanism of GOR is not due to weakness of the LOS or crural diaphragm, rather it is due to transient relaxation of the LOS (TLOSR). ${ }^{12}$ TLOSR is the major mechanism of GOR in normal subjects and patients with mild to moderate gastro-oesophageal reflux disease (GORD). ${ }^{134}$ It is hypothesised that TLOSR is also the predominant mechanism of GOR in patients with moderate to severe GORD. ${ }^{5}$ We have recently shown that atropine, despite causing a significant reduction in basal LOS pressure, ${ }^{67}$ reduces GOR in normal subjects and patients with GORD by inhibiting the frequency of TLOSR. The mechanism by which atropine reduces the frequency of TLOSR is not known.

TLOSR is a neural reflex which is mediated through the brain stem. The afferent pathways for this reflex project from receptors in the gastric fundus to the nucleus tractus solitareus and dorsal motor nucleus of the vagus in the medulla. ${ }^{8}$ The efferent pathway for TLOSR is also in the vagus nerve. ${ }^{9}$ As atropine crosses the blood-brain barrier it may exert its anticholinergic effects either peripherally at the level of the myenteric plexus or centrally, at the level of medulla and higher cerebral centres.

We hypothesised that the inhibitory effects of atropine on TLOSR may be mediated at the level of the central nervous system. Methscopolamine bromide (MSB) is a quaternary ammonium anticholinergic agent, which does not cross the blood-brain barrier. ${ }^{10}$ The aim of this study was to compare the effect of MSB, a peripherally acting anticholinergic agent, with atropine on the rate and mechanism of reflux in patients with GORD.

\section{Methods}

SUBJECTS

Studies were performed in 10 volunteers (four men, six women) with GORD. The latter was defined when classic symptoms of GORD were present and there was evidence of mucosal erosions and ulceration on endoscopy. The median age of the volunteers was 42 years (range 24-54). Volunteers with a history of upper gastrointestinal surgery, or other systemic disease known to influence reflux were excluded from the study. Volunteers with known contraindications for anticholinergic medication including asthma, cardiovascular disease, glaucoma, and prostatic hypertrophy were also excluded. None of the volunteers

Abbreviations used in this paper: GOR gastro-oesophageal reflux; GORD, gastro-oesophageal reflux disease; LOS, lower oesophageal sphincter; MSB, methscopolamine bromide; TLOSR, transient lower oesophageal sphincter relaxation. 
$15 \mathrm{~min} /$ water swallow

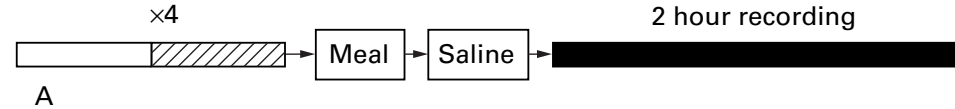

A
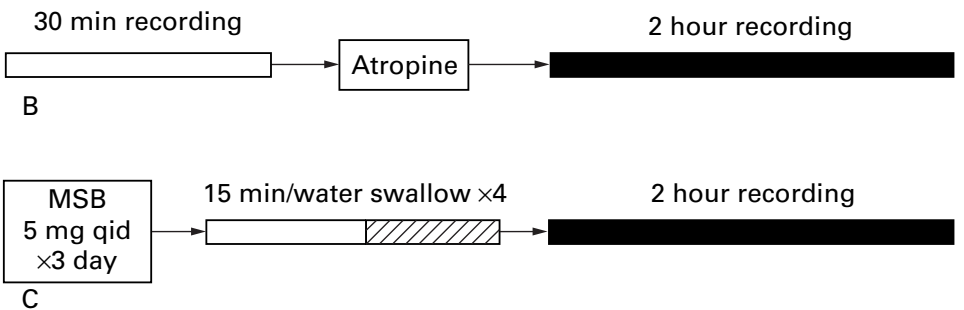

Figure 1 Study design. (A) Baseline recording; (B) recording following atropine infusion; (C) recording following three days of methscopolamine bromide (MSB).

were taking any medication known to affect oesophageal motor function. Proton pump inhibitors were stopped at least five days and $\mathrm{H}_{2}$ antagonists were stopped at least three days prior to the study day. Each volunteer gave written informed consent and the protocol was approved by the University of Virginia Human Investigations Committee.

OESOPHAGEAL MANOMETRY

Oesophageal manometry was performed using a six lumen assembly. A reverse perfused sleeve sensor $^{11}$ monitored LOS pressure. Side holes

Transient LOS relaxation

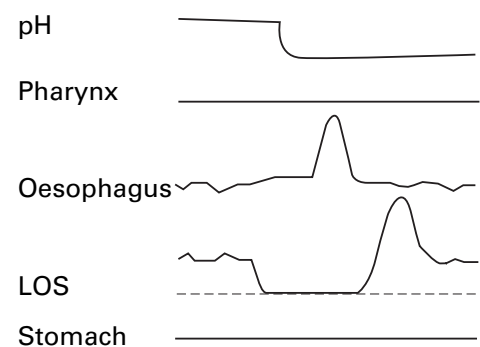

Swallow

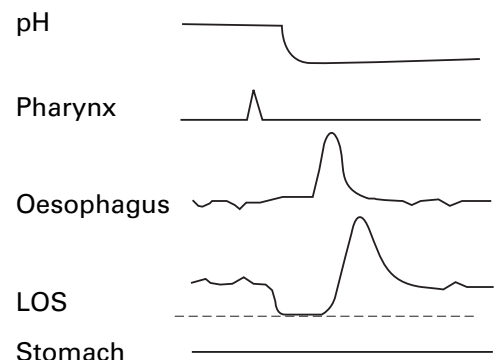

Deep inspiration
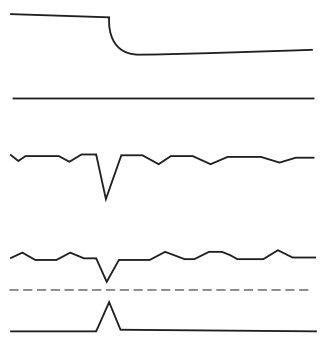

Unknown
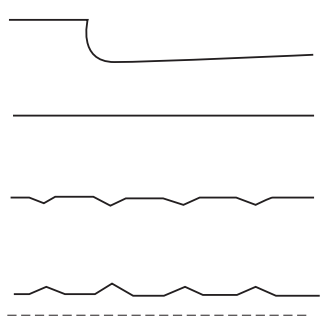

Figure 2 Mechanisms of GOR. TLOSR was defined as a sudden drop in LOS pressure of greater than $5 \mathrm{~mm} \mathrm{Hg}$ in the absence of a pharyngeal contraction and primary oesophageal peristalsis (top left hand panel). Reflux due to deep inspiration was said to occur if there was a drop in oesophageal $p H$ associated with a negative intrathoracic and a positive intragastric pressure (top right hand panel). Reflux due to a swallow occurred when a pharyngeal contraction resulted in a normally conducted oesophageal pressure wave and a brief LOS relaxation associated with a drop in intra-oesophageal $p H$ (bottom left hand panel). Reflux due to an unknown mechanism was when there were no changes in the intra-oesophageal, LOS, and intragastric pressures at the time of reflux (bottom right hand panel). monitored pressure in the pharynx, at three sites in the oesophagus, 2,7 , and $12 \mathrm{~cm}$ above the LOS, and in the gastric fundus. The pharyngeal side hole was water filled but the remaining manometric channels were perfused at $0.5 \mathrm{ml} / \mathrm{min}$ by a low compliance pneumohydraulic capillary infusion pump. Oesophageal $\mathrm{pH}$ was monitored at $5 \mathrm{~cm}$ above the proximal margin of the LOS with an antimony electrode (Synectics Medical, AB, Stockholm, Sweden). The manometric and $\mathrm{pH}$ signals were recorded on a multichannel recorder (Synectics Medical).

STUDY PROTOCOL

Subjects were studied after an eight hour fast. The manometric assembly and $\mathrm{pH}$ electrodes were passed via the nose. A cannula was inserted into the forearm for subsequent infusion of saline or atropine for the first two study periods. The subjects were allowed to accommodate to the assembly for 15 minutes following placement. Each subject then performed four water swallows of a $5 \mathrm{ml}$ bolus. The subjects then ate a standardised $3.8 \mathrm{MJ}$ soft meal. Normal saline was administered intravenously following the ingestion of the meal and recordings were performed for an additional two hours with the subjects in the supine or left lateral position in the bed. On separate days after 30 minutes of control recordings, the subjects received intravenous atropine $(15 \mu \mathrm{g} / \mathrm{kg}$ bolus, $4 \mu \mathrm{g} / \mathrm{kg} / \mathrm{h}$ infusion) and recordings were performed as on day 1 . For the third recording period subjects ingested MSB ( $5 \mathrm{mg}$ orally, four times daily) for three days prior to the recording period. Recordings were obtained for a further two hour period during which the subjects lay quietly in the bed and were asked not to talk (fig 1). Either an assistant or one of the investigators observed the subjects and ensured that they did not sleep during the monitoring period. During atropine infusion the blood pressure and pulse rate were recorded every 15 minutes and the subjects were asked about symptoms such as blurred vision and dry mouth. The saline, atropine, or MSB were given on separate days at least one week apart.

\section{DATA ANALYSIS}

Basal LOS pressure was measured at end expiration and referenced to intragastric pressure; visual mean measurements were taken at five minute intervals and an overall mean for the 120 minute recording period was calculated.

Acid reflux was defined as a drop in $\mathrm{pH}$ of at least two pH units. The onset of the drop in oesophageal $\mathrm{pH}$ was used as the reference time for analysis of the motor events associated with reflux. For each reflux episode the mechanism of reflux was determined from the pattern of LOS pressure, pharyngeal activity, oesophageal body activity, and increases in intragastric pressure (straining). TLOSRs were defined according to the previously published criteria. ${ }^{3}{ }^{12}$ Figure 2 shows the various mechanisms of reflux detected in this study. Primary peristalsis in response to wet swallows was analysed for peristaltic velocity and mean 


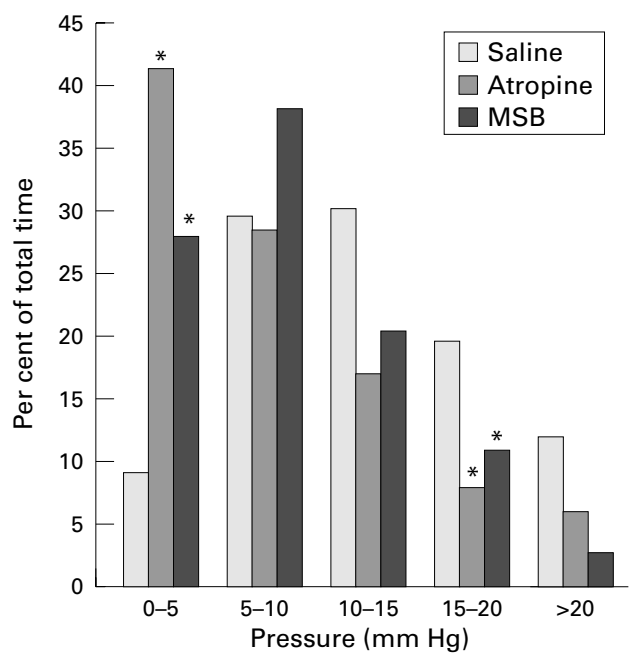

Figure 3 Effect of atropine and MSB on basal LOS pressure. Data are grouped in $5 \mathrm{~mm} \mathrm{Hg}$ pressure intervals and expressed as percentage of total time. ${ }^{*} p<0.05$ versus saline.

amplitude of contraction at the proximal and distal ports.

Data were analysed using the paired $t$ test and Mann-Whitney test. All data are presented as mean (SEM). A p value of less than 0.05 was judged to indicate statistical significance.

\section{Results}

BASAL LOS PRESSURE

Atropine and MSB reduced basal LOS pressure, from $12.6(0.17) \mathrm{mm} \mathrm{Hg}$ to $7.9(0.17) \mathrm{mm}$ $\mathrm{Hg}$ and from $12.6(0.17) \mathrm{mm} \mathrm{Hg}$ to $8.7(0.15)$ $\mathrm{mm} \mathrm{Hg}$ respectively $(\mathrm{p}<0.001$ for both comparisons). Atropine increased the proportion of time LOS pressure was less than $5 \mathrm{~mm} \mathrm{Hg}$ from $9.0(5.2) \%$ to $41(9.4) \%(\mathrm{p}<0.005)$. MSB increased the proportion of time the basal LOS pressure was less than $5 \mathrm{~mm} \mathrm{Hg}$ from 9.0 $(5.2) \%$ to $28 \quad(7.3) \% \quad(\mathrm{p}<0.005) \quad($ fig 3$)$. Atropine and MSB caused a concomitant reduction in the time LOS pressure was between 15 and $20 \mathrm{~mm} \mathrm{Hg}$.

TRANSIENT LOS RELAXATION

Atropine reduced the number of TLOSR episodes versus those occurring with saline (from $8.1(0.56)$ to $2.8(0.55), \mathrm{p}<0.002)$ and MSB (from 7.5 (0.59) to $2.8(0.55), \mathrm{p}<0.005)$. MSB had no effect on the number of TLOSR episodes versus those occurring during saline infusion (7.5 (0.59) versus $8.1(0.56), \mathrm{p}=0.69)$ (fig 4). During saline infusion there were 81 TLOSR episodes of which 32 (39.5\%) were associated with reflux. During atropine infusion there were 28 TLOSR episodes of which nine $(32 \%)$ were associated with reflux. There were 75 TLOSR episodes during the MSB study periods of which $30(40 \%)$ were associated with reflux. There was no significant difference in the percentage of TLOSRs associated with reflux during the three study periods ( $\mathrm{p}>0.05$ for all comparisons).

GASTRO-OESOPHAGEAL REFLUX

Atropine reduced the number of reflux episodes versus saline $(7.0(0.63)$ to $2.0(0.43)$, $\mathrm{p}<0.004)$ and versus MSB (4.9 (0.60) to 2.0
(0.43), p<0.04). MSB had no effect on the number of reflux episodes versus saline (7.0 (0.63) versus $4.9(0.60), p=0.26$ ) (fig 4$)$. Overall, the predominant mechanism of reflux was TLOSR during all three recording periods. Atropine reduced the number of reflux episodes due to TLOSR versus saline (3.2 (0.42) to $0.9(0.30), \mathrm{p}<0.004)$ and versus MSB (2.9 (0.54), p<0.05). MSB, however, had no effect on the number of reflux episodes due to TLOSR versus saline $(3.2(0.43)$ versus 2.9 $(0.54), p=0.85)$. During saline infusion there were 70 episodes of reflux recorded in the 10 subjects of which $32(46 \%)$ occurred during TLOSR (fig 5). During atropine infusion there were 20 episodes of reflux of which nine (45\%) occurred during TLOSR; while on MSB 49 episodes occurred of which $29(59 \%)$ occurred during TLOSR. There was no significant difference in the percentage of reflux occurring during TLOSR for the three study periods ( $p>0.05$ for all comparisons).

Cholinergic blockade also reduced the number of reflux episodes due to swallow induced LOS relaxation (fig 5). During saline infusion there were 21 episodes of reflux occurring during swallow induced LOS relaxation versus three episodes each during atropine infusion and the MSB period.

EFFECT ON PRIMARY SWALLOW INDUCED OESOPHAGEAL CONTRACTIONS

MSB significantly reduced the oesophageal contraction amplitude at both the proximal and distal ports as well as increased the velocity of peristalsis compared with saline (table 1). Wet swallows were performed prior to atropine infusion; its effects on primary peristalsis were therefore not measured.

SYSTEMIC AND OESOPHAGEAL ANTICHOLINERGIC EFFECTS

There were no significant differences in the type or total number of anticholinergic side effects noted by the subjects while taking atropine or MSB. The number of subjects experiencing any anticholinergic side effect was the same during atropine infusion or while taking MSB (table 2).

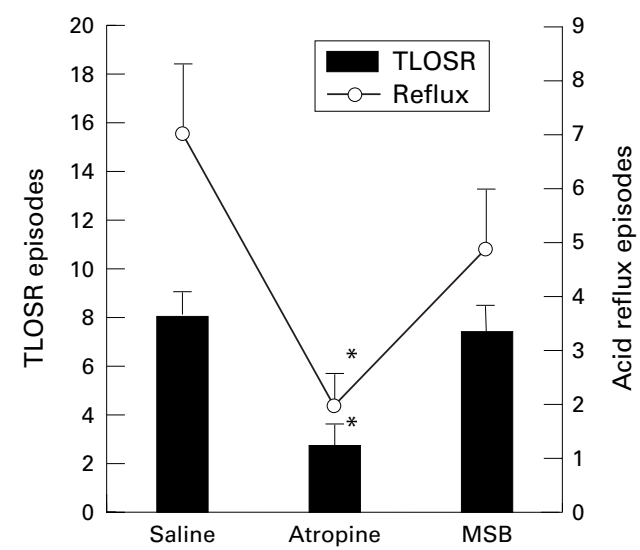

Figure 4 Effects of atropine and MSB on the mean number of reflux episodes and TLOSRs during the two hour recording period. ${ }^{\star} p<0.05$. 


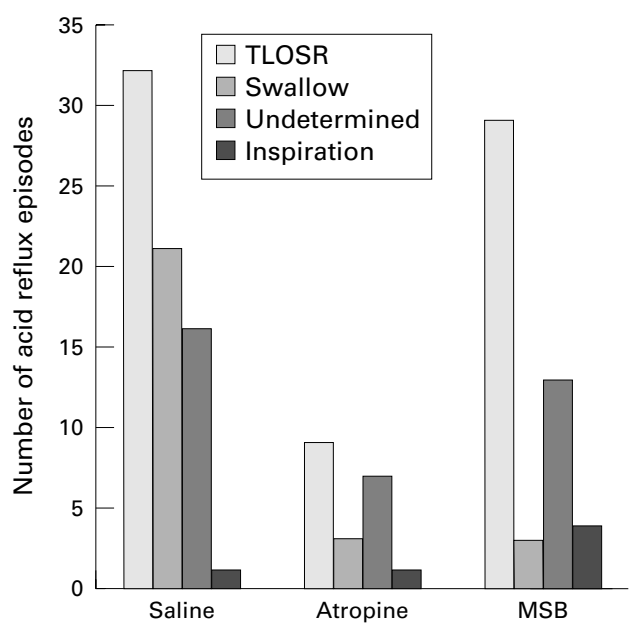

Figure 5 Mechanism of reflux during saline, atropine, and MSB periods.

Table 1 Effect of MSB on primary peristalsis

\begin{tabular}{lcl}
\hline & Saline & MSB \\
\hline Proximal amplitude (mm Hg) & $82(3.6)$ & $58(4.8)^{\star}$ \\
Distal amplitude (mm Hg) & $102(6.1)$ & $86(7.1)^{\star}$ \\
Distal velocity $(\mathrm{cm} / \mathrm{s})$ & $2.5(0.13)$ & $2.0(0.12)^{\star}$
\end{tabular}

Data expressed as mean (SEM); ${ }^{\star} \mathrm{p}<0.05$ versus saline.

Table 2 Incidence of anticholinergic side effects in subjects while taking atropine or MSB

\begin{tabular}{lccl}
\hline & Atropine (n) & MSB (n) & $p$ Value \\
\hline Headache & 1 & 3 & NS \\
Blurred vision & 3 & 0 & NS \\
Dry mouth & 6 & 6 & NS \\
Dry skin & 2 & 5 & NS \\
Decreased sweating & 0 & 2 & NS \\
Palpitations & 3 & 0 & NS \\
Dizziness & 1 & 2 & NS \\
Total number of side effects & 16 & 18 & NS \\
Any side effect & 8 & 8 & NS \\
\hline
\end{tabular}

$\mathrm{n}$, number of subjects with listed side effect; NS, not significant.

\section{Discussion}

Atropine has previously been shown to decrease GOR through a reduction in the TLOSR in healthy subjects and patients with GORD.${ }^{6}$ However, it was unclear whether the mechanism of action was through central or peripheral cholinergic blockade. Selective peripheral cholinergic blockade with MSB did not reduce GOR, TLOSR, or GOR due to TLOSR in patients with GORD. Therefore, we may conclude that atropine induced inhibition of GOR and TLOSR is most likely mediated through a central cholinergic blockade.

Could the lack of inhibition of GOR and TLOSR by MSB be due to insufficient dosage to achieve peripheral cholinergic blockade? The dose chosen in our study was higher than initially recommended to achieve an anticholinergic effect ${ }^{10}$ and most subjects noted anticholinergic side effects while on MSB or atropine. More convincingly, MSB administration reduced the mean basal LOS pressure to almost the same degree as seen with atropine. Finally, MSB reduced the amplitude of primary peristalsis as has been shown previously with atropine. ${ }^{13}$

In a previous study of patients with GORD, the frequency of GOR was reduced by near abolition of both TLOSR induced reflux and swallow induced reflux. ${ }^{7}$ In this study we found that reflux occurring during swallow induced LOS relaxation was notably decreased with atropine which is in accordance with the previous published study. We found that in this study, similarly to atropine, MSB also reduced the swallow mediated reflux. However, while atropine significantly reduced the overall number of TLOSR episodes and GOR due to TLOSR compared with placebo and MSB, the relative frequency of GOR due to TLOSR was not significantly different. This most likely reflects the heterogeneous mechanisms of reflux in patients with GORD ${ }^{14}$ and differences in the two GORD populations studied.

The central neural pathways mediating TLOSR have not been clearly defined, but are thought to reside in the brain stem. Afferent stimuli from the gastric fundus travel to the sensory nucleus in the medulla, the nucleus tractus solitareus. A programmed set of events in the brain stem occurs in the region of the dorsal vagal nucleus and the nucleus ambiguous, which then mediates the efferent signals for TLOSR. ${ }^{8}$ The central anticholinergic effect of atropine most likely occurs in this region of the brain stem. In support of this concept is the observation that atropine reduces the oral phase of swallowing in response to electrical stimulation of the superior laryngeal nerve in the opossum, suggesting that atropine acts at the level of the central nervous system to block the swallow reflex. ${ }^{14}$ Furthermore, Berger reported that cholinergic receptors are present in the swallowing centre in the brain stem and muscarinic blockade inhibits the oesophageal phase of swallowing. ${ }^{15}$

Atropine was recently reported to inhibit gastric distension induced TLOSR and pharyngeal receptor mediated LOS relaxation. ${ }^{17}$ The mechanism of the effect of atropine on gastric distension induced TLOSR could be mediated through its inhibitory effect on gastric fundic tone. It was also proposed that inhibition of both gastric distension and pharyngeal receptor mediated LOS relaxation could be mediated through a central cholinergic blockade. ${ }^{16}$ The data from the current study support the existence of a central mechanism of TLOSR which is sensitive to cholinergic blockade.

Our results are in agreement with the recently reported effects of hyoscine bromide, a peripherally acting anticholinergic agent, on GOR and TLOSR in normal subjects published in abstract form. ${ }^{17}$ Lidums et al also found that peripheral cholinergic blockade with hyoscine had no effect on GOR and TLOSR. Hyoscine bromide, similar to MSB and atropine, had inhibitory effects on the oesophageal contraction amplitude. ${ }^{17}$

We have previously shown that despite decreasing basal LOS pressure, non-selective cholinergic blockade with atropine actually decreases GOR by decreasing TLOSR in healthy subjects and in patients with GORD. This study further elucidates the mechanism of action of atropine by showing that it occurs through a central cholinergic blockade. Pharmacological reduction of GOR by inhibition of 
TLOSR with anticholinergic agents is an attractive therapeutic option to treat GORD by reversing its primary pathophysiological derangement. However, atropine and MSB reduce the salivary flow and reduce the amplitude of primary oesophageal peristalsis, both of which are important for normal oesophageal acid clearance. Therefore this class of compounds is contraindicated in reflux disease. However, whether it is possible to design a selective centrally acting anticholinergic compound that may inhibit TLOSR without any deleterious effects on saliva and oesophageal contractions remains to be seen.

This study was supported by NIH grant RO3 DK 52094-01 SRC C and by a restricted grant from Astra Merck Pharmaceuticals

1 Dent J, Dodds WJ, Friedman RH, et al. Mechanism of gastroesophageal reflux in recumbent asymptomatic human troesophageal reflux in recumbent asy

2 Dodds WJ, Dent J, Hogan WJ, et al. Mechanism of gastroesophageal reflux in patients with reflux esophagitis. gastroesophageal reflux in patients

3 Dent J, Holloway RH, Toouli J, et al. Mechanism of lower oesophageal incompetence in patients with symptomatic gastro-oesophageal reflux. Gut 1988;29:1020-8.

4 Penagini R, Schoeman M, Holloway R, et al. Mechanisms of reflux in ambulant patients with reflux esophagitis [abstract]. Gastroenterology 1994;106:159A.

5 Mittal RK, Balaban DH. Mechanisms of disease: the esophagogastric junction. $N$ Engl F Med 1997;336:924-32.
6 Mittal RK, Holloway RH, Dent J. Effect of atropine on the frequency of lower esophageal sphincter relaxation in norfrequency of lower esophageal sphincter relaxation

7 Lidums I, Holloway RH, Checklin H, et al. Effect of atropine on gastro-oesophageal reflux and transient lower oesophageal sphincter relaxation in patients with gastrooesophageal reflux disease. Gut 1998;43:12-16.

8 Mittal RK, Holloway RH, Penagini R, et al. Transient lower esophageal sphincter relaxation. Gastroenterology 1995;109: $601-10$.

9 Holloway RH, Wyman, Dent J. Failure of transient lower oesophageal relaxations in response to gastric distension in patients with achalasia: evidence for neural mediation of patients with achalasia: evidence for neural mediation of transient lower

10 Taunton, MA. Drug information for the health care professional. United States Pharmacopeia, 1995

11 Sivri B, Mittal RK. Reverse perfused sleeve: an improved device for measurement of sphincteric function of crural diaphragm. Gastroenterology 1992;101:962-9.

12 Holloway RH, Penagini R, Ireland AC. Criteria for the objective definition of transient lower esophageal sphincter relaxation. Am f Physiol 1995;268:G128-33.

13 Dodds WJ, Dent J, Hogan WJ, et al. Effect of atropine on esophageal motor function in humans. Am F Physiol 1981; 81:G290-6.

14 Penagini R, Schoeman MN, Dent J, et al. Motor events underlying gastro-oesophageal reflux in ambulant patients with reflux oesophagitis. F Neurogastroenterol Motil 1996;8: $131-41$

15 Berger D. Muscarinic activation of rhombencephalic neurones controlling oesophageal peristalsis in the rat. Neuropharmacology 1984;23:1451-64.

16 Mittal RK, Chiareli C, Liu J, et al. Atropine inhibits gastric distension and pharyngeal receptor mediated lower distension and pharyngeal receptor mediated lower
oesophageal sphincter relaxation. Gut 1997;41:285-90.

17 Lidums I, Bermingham, Holloway RH. Effect of hyoscine butylbromide on gastroesophageal reflux and lower esophageal sphincter in healthy volunteers [abstract]. Gastroenterology 1997;112:A778. 